\title{
Special issue on selected papers from CAIP 2015
}

\author{
George Azzopardi ${ }^{1} \cdot$ Nicolai Petkov ${ }^{2}$
}

Published online: 3 December 2016

(C) Springer-Verlag Berlin Heidelberg 2016

CAIP (International Conference on Computer Analysis of Images and Patterns; http://caip.eu.org) is a series of biennial international conferences devoted to all aspects of computer vision, image processing and analysis, pattern recognition and related fields. The scientific program of the conference consists of keynote addresses and contributed papers presented in single-track oral and poster sessions. CAIP has been held in: Berlin (1985), Wismar (1987), Leipzig (1989), Dresden (1991), Budapest (1993), Prague (1995), Kiel (1997), Ljubljana (1999), Warsaw (2001), Groningen (2003), Paris (2005), Vienna (2007), Münster (2009), Seville (2011), York (2013) and Malta (2015). The next CAIP conference will be in Ystad (Sweden), 2017.

CAIP enjoys a high international visibility and attracts participants from all over the world. CAIP 2015 was held in Valletta, Malta, from September 2, 2015, to September 4, 2015. It aimed to extend the scope of the series by allowing also submissions in pattern recognition of non-image data, machine learning and brain-inspired computing. Each submission was reviewed by at least three members of the International Program Committee, and only high-quality papers were selected for inclusion in the proceedings published by Springer in Lecture Notes on Computer Science, Vol 9256 and 9257.

This special issue contains 18 selected papers from the scientific program of CAIP 2015, which were substantially extended and underwent a thorough reviewing. Two of the keynote speakers in CAIP 2015 Bart ter Haar Romenij and Patrizio Campisi together with their co-authors contributed the papers "Brain-Inspired Algorithms for Retinal Image Analysis" and "EEG Signal Preprocessing for Biometric Recognition," respectively. The other papers cover mainly topics in image processing, (biomedical) image analysis, motion analysis, shape recovery, biometrics and graph-based representations for pattern recognition.

We like to thank Rahul Sukthankar, Editor-in-Chief of Machine Vision and Applications journal, for giving us the opportunity to edit this special issue and Cherry Place from the editorial office for her support in managing the issue. Our thanks also go to Deepika Sureshkumar, production editor of Springer, for her support and the reviewers for their valuable comments.
George Azzopardi

george.azzopardi@um.edu.mt

Nicolai Petkov

n.petkov@rug.nl

1 University of Malta, Msida, Malta

2 University of Groningen, Groningen, The Netherlands 